\title{
Vestibular Schwannoma with Contralateral Facial Nerve Palsy: A False Localizing Sign
}

\author{
Mukesh K. Bhaskar $^{1} \quad$ Rakesh Kumar $^{1}$ Sunil K. Singh ${ }^{1} \quad$ M. Meel ${ }^{2}$ \\ ${ }^{1}$ Department of Neurosurgery, King Gerorge's Medical University, \\ Lucknow, Uttar Pradesh, India \\ 2 Department of Pathology, RNT Medical College, Udaipur, \\ Rajasthan, India \\ Indian J Neurosurg 2017;6:141-143.
}

Address for correspondence Mukesh K. Bhaskar, MS, Department of Neurosurgery, King Gerorge's Medical University, Lucknow, UP, India 226003 (e-mail: drmannu84@gmail.com).

\begin{abstract}
Keywords

- vestibular schwannoma

- facial nerve paresis

- false localizing sign

Vestibular schwannomas are the most common cerebellopontine angle tumors. These tumors commonly present with ipsilateral dysfunction of acoustic, vestibular, trigeminal, and facial nerves. Vestibular schwannoma with involvement of contralateral facial nerve is very unusual, and whenever present, it is considered as a false localizing sign. It seems that displacement and distortion of the brainstem by the large mass lesion may lead to this atypical presentation. We report a case of vestibular schwannoma with contralateral abducens and facial nerve paresis.
\end{abstract}

\section{Introduction}

Vestibular schwannomas are the most common cerebellopontine angle tumors. These tumors commonly present with ipsilateral dysfunction of acoustic, vestibular, trigeminal, and facial nerves. ${ }^{1}$ Presentation of the vestibular schwannoma with contralateral facial nerve dysfunction is quite uncommon, and the contralateral facial paresis has been mentioned as a false localizing sign for posterior fossa and cerebellopontine angle tumors. ${ }^{2-4}$ Among 287 patients with vestibular schwannoma who underwent surgery at our institute during past 8 years, we encountered one 1 patient with this unusual presentation.

\section{Case Report}

A 35-year-old woman presented with history of vertigo, progressive left-sided loss hearing, and gait ataxia for 1 year, and headache, diplopia, and progressive asym-metry of the face for 2 months. Neurologic examination showed bilateral grade 3 papilledema, decreased left-sided corneal sensation, left-sided sensory-neural hearing loss, left-sided delayed taste sensation, deviation of the face toward left side (House-Brackmann grade 3; right-sided facial palsy), and right-sided sixth nerve paresis ( $\boldsymbol{- F i g . 1}$ ). Her cerebellar tests were abnormal on the left side. Magnetic resonance imaging
(MRI) showed a large $((39 \times 37 \times 39 \mathrm{~mm})$ left cerebellopontine angle mass that appears hypointense on T1W1 images and hyperintense on T2W1 images with heterogenous enhancement on gadolinium administration (-Fig. 2A-C). Hydrocephalus with periventricular lucency and distortion of the brainstem were also seen. The radiologic impression was suggestive of vestibular schwannoma and the patient was prepared for surgery.

A left retromastoid suboccipital craniectomy with near total excision of the lesion was performed with preservation of facial nerve by using intraoperative nerve monitoring. Postoperative recovery was uneventful, and postoperative computed tomographic (CT) scan showed complete excision of the lesion. Histopathologic examination (HPE) of the specimen confirmed the preoperative diagnosis of vestibular schwannoma. Postoperatively, double vision subsided but right facial nerve paresis showed no improvement.

\section{Discussion}

False localizing signs are unexpected neurologic deficits based on anatomic location and pathophysiologic processes. ${ }^{5}$ Cranial nerve palsies, hemiparesis, sensory features, and muscle atrophy may all occur as false localizing signs. ${ }^{6}$ Review of literature showed various cranial nerve dysfunction as a false localizing sign, including abducens nerve received

September 3, 2016

accepted

January 31, 2017

published online

May 19, 2017
DOI https://doi.org/

10.1055/s-0037-1602753. ISSN 2277-954X. (c) 2017 Neurological Surgeons' Society of India
License terms

(요 (1) $\Theta$ 


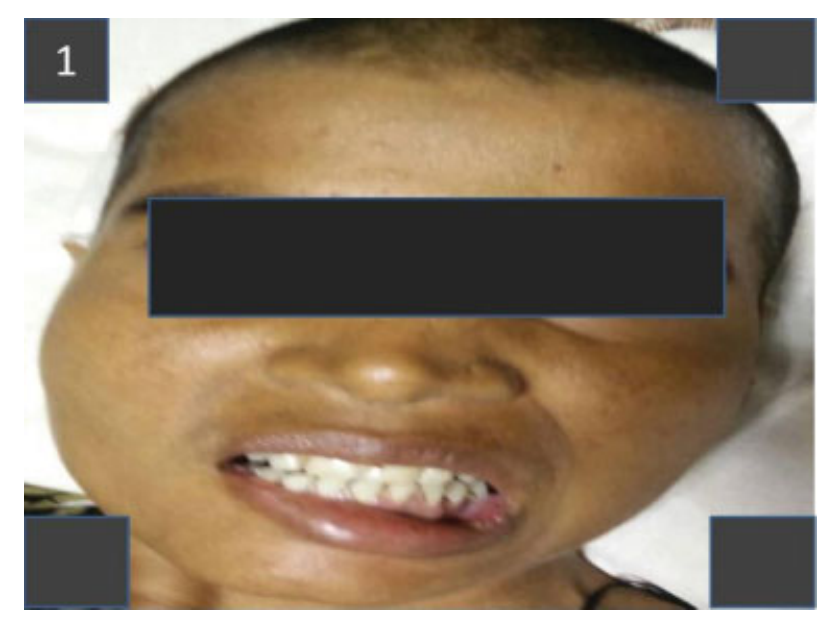

Fig. 1 Bilateral grade 3 papilledema, decreased left-sided corneal sensation, left-sided sensory-neural hearing loss, left-sided delayed taste sensation, deviation of the face toward left side, and rightsided sixth nerve paresis.

(most common), trigeminal nerve, facial nerve, vestibulocochlear nerve, and oculo-motor nerve. ${ }^{2-7}$ Multiple cranial nerve dysfunction presenting as false localizing signs is very rare. Concurrent involvement of the trigeminal sensory, abducens, and facial nerves as false localizing signs was reported by Ro et al. ${ }^{2}$ Involvement of both the facial and vestibulocochlear nerves as false localizing signs was reported by Paillas et al. ${ }^{3}$ Our case is distinct from these cases in that we report dysfunction of the contralateral abducens and facial nerves as false localizing signs.

Multiple cranial nerve palsy associated with cerebellopontine angle and posterior fossa tumors has been attributed to different factors such as the tumor size, raised intracranial pressure (ICP), compression and distortion of the brainstem, angulations and distortion of the nerve roots, and the relationships of cranial nerves and nearby blood vessels. The direction of displacement is determined by the anatomic variations of the bony surface and surrounding rigid structures. ${ }^{8}$ According to Paillas et al, tumor size (large) and nature (firm) were responsible for false localizing signs of cranial nerve. ${ }^{3}$

The abducens nerve palsy is most common cranial nerve dysfunction presented as false localizing sign. ${ }^{8}$ The abducens nerve dysfunction in cases of raised ICP was possibly caused by a posterior shift of the brainstem and stretching of this nerve in its vertical course in a direction opposite to the brain displacement. ${ }^{5}$ The abducens nerve can also be entrapped or strangulated by the branches of the basilar artery when the pons and medulla are distorted by the posterior fossa tumor. ${ }^{9}$ However, it is difficult to determine which mechanism contributed to the clinical presentation of our case (raised ICP and brainstem distortion both were present).

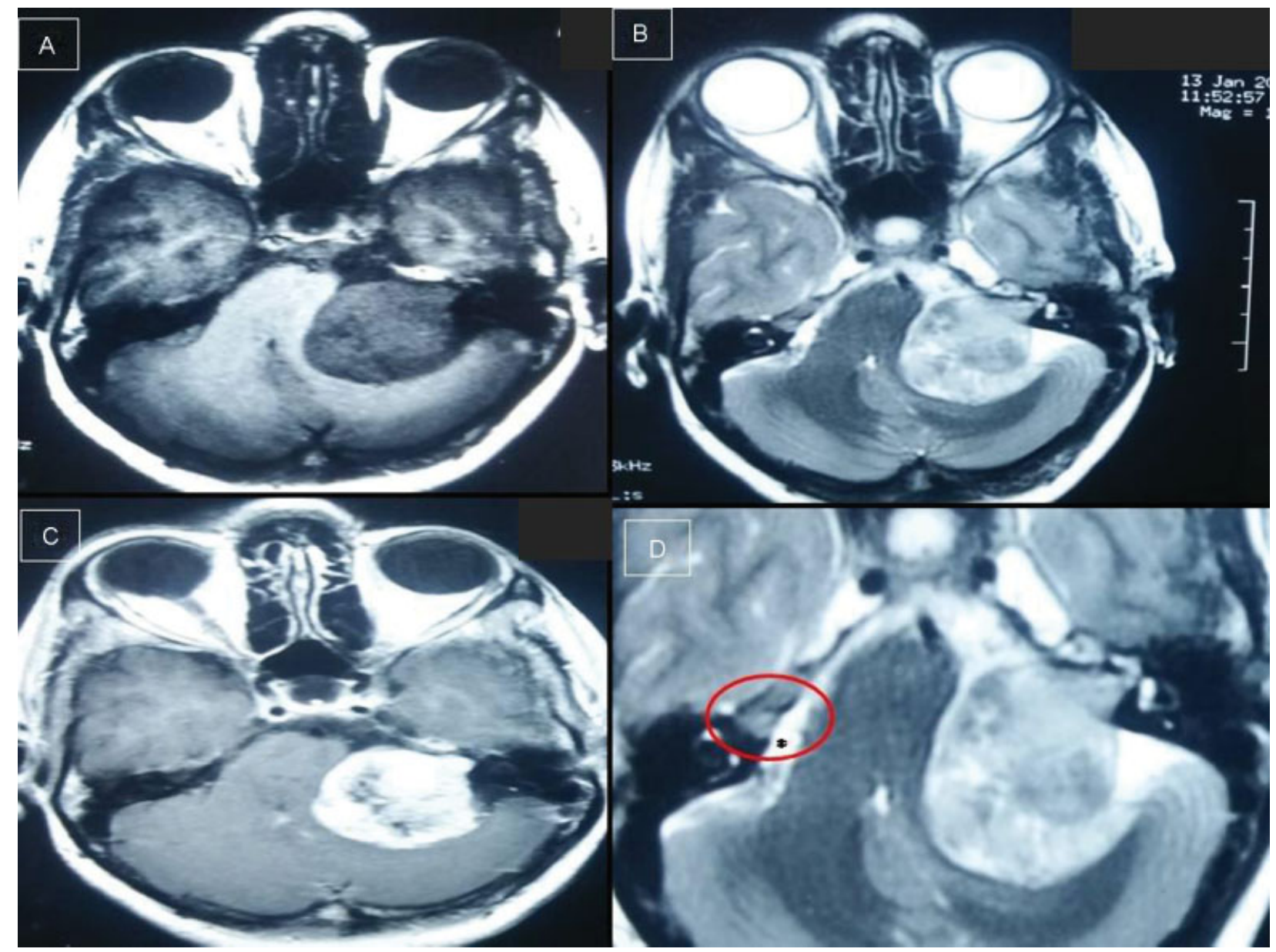

Fig. 2 MRI showing a large $(39 \times 37 \times 39 \mathrm{~mm})$ left cerebellopontine angle mass. 
The facial nerve proper and intermediate and vestibulocochlear nerves leave the brainstem and pass through the internal auditory meatus along a bony canal, which may make them subject to compression on the margins of the meatus when the brainstem is displaced. ${ }^{2}$ The nervus intermedius is a small bundle that usually leaves the pons closer to the vestibulocochlear nerve than facial nerve proper and runs between them across the cerebellopontine angle cistern, moving closer to facial nerve proper as it enters the facial canal. In our case only the facial nerve proper but not intermediate or vestibulocochlear nerve is involved as a false localizing sign. The variable vessel-facial nerve and vesselvestibulocochlear nerve relationships outside and inside the internal meatus and the nearby blood vessels might compress or strangulate the nearby facial nerve only when the brainstem is distorted. ${ }^{10}$ In our case, there is a wellmaintained cerebrospinal fluid (CSF) subarachnoid space in right CP angle cistern (-Fig. 2D asterisk) with no MRI evidence of compression of distorted brainstem onto the adjacent petrous bone (-Fig. 2D). Even the right seventh/ eight nerve complex also appears intact. Hence a high possibility of a vascular event in the form of ischemia or strangulation of the nerve by a vessel loop explains the nonrecovering facial nerve deficit. However, cranial nerve anatomy can be better delineated with special MRI sequences such as FIESTA/CISS (fast imaging employing steady-state acquisition/constructive interference in steady state) (not done in our case). Reports of false localizing seventh nerve palsies suggest that they most often occur at the same time as, or after, the development of sixth nerve palsies. ${ }^{2,5,11}$ In our case both palsies were involved at the time of presentation, and hence the exact cause cannot be ascertained.

\section{Conclusion}

Contralateral facial palsy is an extremely rare presentation of vestibular schwannomas even with brainstem distortion. The causative mechanism is most probably displacement and distortion of the brainstem, with or without a vascular pathology. A number of factors are responsible for false localizing sign such as tumor size and consistency raised ICP, compression and distortion of the brainstem, angulations and distortion of the nerve roots, and the relationships of cranial nerves and nearby blood vessels.
Source(s) of Support

The study was supported by Department of Neurosurgery, King George's Medical University, Lucknow, Uttar Pradesh, India.

\section{Presentation at a Meeting}

None.

\section{Conflict of Interest}

None.

\section{Acknowledgments}

The authors sincerely thank Prof. B. K. Ojha, Prof. Anil Chandra, Dr. C. Srivastava, Dr. Manish Jaiswal, and Dr. Somil Jaiswal for their constant support and help in preparation of the manuscript including submission procedures.

\section{References}

1 Matthies C, Samii M. Management of 1000 vestibular schwannomas (acoustic neuromas): clinical presentation. Neurosurgery 1997;40(01):1-9, discussion 9-10

2 Ro LS, Chen ST, Tang LM, Wei KC. Concurrent trigeminal, abducens, and facial nerve palsies presenting as false localizing signs: case report. Neurosurgery 1995;37(02):322-324, discussion 324-325

3 Paillas JE, Pellet W, Janny P, Tournilhac M, Komminoth J. L'atteinte contro-latérale des nerfs crâniens au cours des tumeurs de la fosse postérieure. Rev Neurol (Paris) 1969;121(04):452-464

4 Maurice-Williams RS. Multiple crossed false localizing signs in a posterior fossa tumour. J Neurol Neurosurg Psychiatry 1975; 38(12):1232-1234

5 Collier J. The false localizing signs of intracranial tumor. Brain 1904;27:492-508

6 Larner AJ. False localising signs. J Neurol Neurosurg Psychiatry 2003;74(04):415-418

7 Marshman LAG, Polkey CE, Penney CC. Unilateral fixed dilation of the pupil as a false-localizing sign with intracranial hemorrhage: case report and literature review. Neurosurgery 2001;49(05):1251-1255, discussion 1255-1256

8 O'Connell JEA. Trigeminal false localizing signs and their causation. Brain 1978;101(01):119-142

9 Cushing H. Strangulation from nervi abducentes by lateral branches of the basilar artery in cases of brain tumour. Brain 1910;33:204-235

10 Lang J. Facial and vestibulocochlear nerve, topographic anatomy and variations. In: Samii M, Jannetta PJ, eds. The Cranial Nerves. Berlin, Germany: Springer-Verlag; 1981:363-377

11 Davie C, Kennedy P, Katifi HA. Seventh nerve palsy as a false localising sign. J Neurol Neurosurg Psychiatry 1992;55(06): 510-511 